\title{
Effect of Mesh on Springback in 3D Finite Element Analysis of Flexible Microrolling
}

\author{
Feijun Qu, Zhengyi Jiang, and Haina Lu \\ Faculty of Engineering and Information Sciences, University of Wollongong, Wollongong, NSW 2522, Australia \\ Correspondence should be addressed to Zhengyi Jiang; jiang@uow.edu.au
}

Received 10 June 2015; Accepted 9 August 2015

Academic Editor: Sutasn Thipprakmas

Copyright ( 2015 Feijun Qu et al. This is an open access article distributed under the Creative Commons Attribution License, which permits unrestricted use, distribution, and reproduction in any medium, provided the original work is properly cited.

\begin{abstract}
In flexible microrolling, springback in thickness direction is a critical indicator to determine the forming quality. Accurate prediction of springback is one of the significant aspects in the finite element analysis of flexible microrolling. Meshing is a step of great importance in finite element analysis of manufacturing process as it directly determines the accuracy of the FEA results as well as the requested computational time. This paper presents a numerical study on revealing the mesh effects on the accuracy of springback estimation utilising ABAQUS/Standard for modelling and analyses. Two types of meshes with six mesh sizes for each mesh type are considered in this study and the optimal mesh type and mesh size have been found to obtain accurate value of springback while saving as much computational time as possible.
\end{abstract}

\section{Introduction}

The finite element analysis (FEA) is a powerful computational tool for performing various scientific and engineering analyses. The use of mesh generation techniques for dividing a complex problem into small elements plays a crucial role in FEA, which determines the accuracy of the FEA results and the requested computational time.

Many researchers have investigated the effects of mesh size on the accuracy of numerical simulation results. Shi et al. [1] used both coarse mesh and fine mesh to study the mesh size effect on numerical results of blast wave propagation and interaction with structures. It was observed that a coarse mesh induced errors in estimation of the positive reflected peak pressure in blast scenario. Choi and Kwak [2] investigated the influence of mesh size on nonlinear finite element analysis of the behaviour of reinforced concrete structure. In their study, the calculated value with relatively small mesh size was found to better approximate the experimental data and the numerical error was hardly noticeable. Cojocaru et al. [3] discussed the mesh size effect on stresses and strains in finite element analysis of a gear housing. Six different mesh sizes were performed in the FEA simulations. The results revealed that the mesh density had high significance to the stresses and insignificance to the strains, where the difference between the maximum von Mises stresses reached $20 \%$. Kim et al. [4] presented a finite element study of the mesh density effect on the mechanical behaviour prediction of a pyramidal unit cell. The case studies of the mechanical behaviours of the unit cell under compression loading such as peak stress, effective modulus, and deformed geometry with various mesh conditions indicated that higher mesh density offered more accurate results undoubtedly. Smith and Hobbs [5] assessed the effect of mesh size on the behaviour of model slopes in a centrifuge via two aspect ratios of 0.5 and 1. Discrepancy between the simulation outcomes and the experimental results was noted when the coarser mesh with aspect ratio of 0.5 was used. Lai et al. [6] conducted an analysis of the effect of mesh density on the numerical simulation results of fluid-structure interaction. It was demonstrated in their research that calculation errors between simulation results and experimental data reduced according to the mesh density increasing from 2 to 3 . Koslan et al. [7] studied the effect of mesh sizing toward deformation result in simulation for blast loading application and they mentioned that the percentage of error for deformation result decreased due to the sufficient refinement of finite element grid, which however cost more computational time. Candal et al. [8] focused on investigating the impact of mesh density on injection molding simulation results. It was found 
that when the number of elements increased, the time for obtaining simulation results also increased, but with a better convergence in the values obtained.

From the previous related literature, it can be seen that the finite element models with fine mesh yield highly accurate results but may take longer computational time. Small element size will increase the FE model's complexity, which is only used when high accuracy is demanded. On the contrary, large element size will simplify the FE model and is extensively used in order to provide a quick and rough estimation of designs.

Moreover, the influence of mesh on springback prediction has also been highlighted in previous research activities. Pham et al. [9] established a numerical model to investigate the springback of $U$-shaped parts made of ultrathin stainless steel after deep-drawing with three mesh sizes. Prediction with the finest mesh was found to be closer to the experimental result, but taking the longest CPU time of $168 \mathrm{~h}$. $\mathrm{Xu}$ et al. [10] studied the relationship between the number of the blank mesh sizes and the accuracy and efficiency of springback simulation, taking the U-bending process as an example. They concluded that five elements contacting die radius produced better solution than that of three elements, but with increasing calculation time by $1014.08 \mathrm{~s}$. Eggertsen et al. [11] analysed the springback during drawing bending process and confirmed that better simulation result could be attained using smaller mesh size. Nevertheless, there are few reports concerning flexible microrolling process, especially the springback of the blank along thickness direction. Although Xie et al. $[12,13]$ conducted numerical and experimental investigations on microrolling of stainless steel foil, they concentrated on friction behaviour and evolution of surface roughness during the microrolling process without taking into consideration the springback of the foil in thickness direction and the effect of reduction. On this account, it is consequential to look into the microrolling process numerically from the aspects of springback and inconstant reduction as well as the influence of simulation parameters on the calculated results.

In this study, a 3D flexible microrolling finite element model is established by applying different mesh sizes and types of meshes. The springback of the blank in thickness direction is analysed to reveal the mesh effects on the accuracy of the FEA results and present guidelines for choosing appropriate mesh for finite element analyses of microrolling process.

\section{Numerical Analysis during Flexible Microrolling Process}

As flexible microrolling process involves nonlinear analysis, the solution usually cannot be calculated by solving a single system of equations. Instead, the solution is obtained iteratively by use of the Newton-Raphson method [14].

System of nonlinear algebraic equations can be written as

$$
\psi(\mathbf{a})=\mathbf{P}(\mathbf{a})-\mathbf{Q}=0
$$

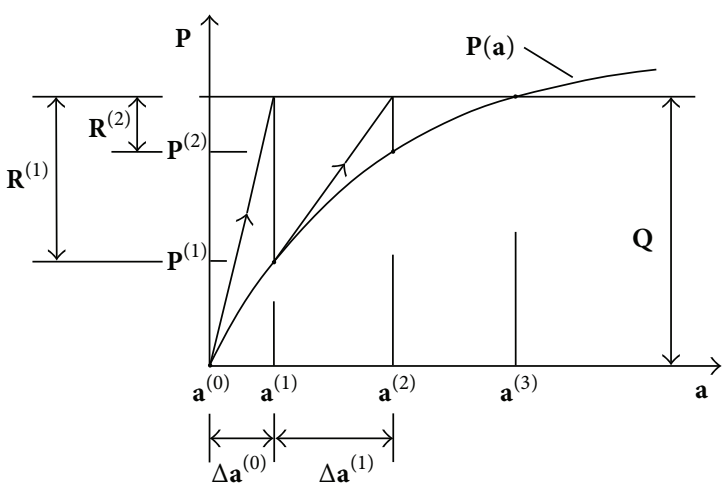

FIGURE 1: Convergence of the Newton-Raphson iteration process.

where $\mathbf{a}$ is the nodal displacement vector and $\mathbf{Q}$ is the nodal load vector in the finite element analysis with an unknown amount of displacement.

If a trial value of $\mathbf{a}^{(n)}$ is found sufficiently close to the correct one but at which $\psi(\mathbf{a}) \neq 0$, the improved trial solution can be obtained by finding

$$
\mathbf{a}^{(n+1)}=\mathbf{a}^{(n)}+\Delta \mathbf{a}^{(n)}
$$

with taking a Taylor expansion in the vicinity of $\mathbf{a}^{(n)}$ and keeping only linear terms [15]

$$
\psi\left(\mathbf{a}^{(n+1)}\right) \equiv \psi\left(\mathbf{a}^{(n)}\right)+\left(\frac{\mathrm{d} \psi}{\mathrm{d} \mathbf{a}}\right)_{n} \Delta \mathbf{a}^{(n)}=0,
$$

where $\mathrm{d} \psi / \mathrm{d} \mathbf{a}$ is the tangent stiffness matrix; namely,

$$
\frac{\mathrm{d} \psi}{\mathrm{d} \mathbf{a}} \equiv \frac{\mathrm{d} \mathbf{P}}{\mathrm{d} \mathbf{a}} \equiv \mathbf{K}_{T}(\mathbf{a}) .
$$

Equations (3) and (4) give

$$
\begin{aligned}
\Delta \mathbf{a}^{(n)} & =-\left(\mathbf{K}_{T}^{(n)}\right)^{-1} \psi^{(n)}=-\left(\mathbf{K}_{T}^{(n)}\right)^{-1}\left(\mathbf{P}^{(n)}-\mathbf{Q}\right) \\
& =\left(\mathbf{K}_{T}^{(n)}\right)^{-1}\left(\mathbf{Q}-\mathbf{P}^{(n)}\right),
\end{aligned}
$$

where $\mathbf{K}_{T}^{(n)}=\mathbf{K}_{T}\left(\mathbf{a}^{(n)}\right), \quad \mathbf{P}^{(n)}=\mathbf{P}\left(\mathbf{a}^{(n)}\right)$.

Since the Taylor expansion equation (3) takes only linear terms, $\mathbf{a}^{(n+1)}$ is still an approximate solution. Iteration is continued until the convergence requirements are met.

Graphically, the convergence of the Newton-Raphson iteration process is illustrated in Figure 1. It can be seen that the difference between the total applied load, $\mathbf{Q}$, and $\mathbf{P}^{(n)}$ can be calculated as

$$
\mathbf{R}^{(n)}=\mathbf{Q}-\mathbf{P}^{(n)},
$$

where $\mathbf{R}^{(n)}$ is the force residual for the iteration [16].

In such a nonlinear problem, it is almost impossible to have $\mathbf{R}^{(n)}$ equal zero, so a tolerance value is set for comparison. If $\mathbf{R}^{(n)}$ is less than the tolerance value, $\mathbf{Q}$ and $\mathbf{P}^{(n)}$ are in equilibrium, and $\mathbf{a}^{(n+1)}$ is a valid equilibrium configuration for the model under the applied load. 


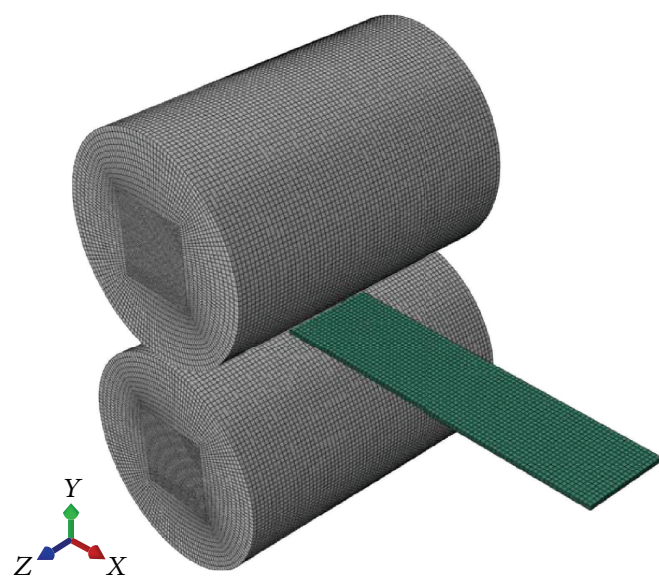

FIGURE 2: FE model of flexible microrolling.

However, before the solution is accepted, $\Delta \mathbf{a}^{(n)}$ is also checked, which is small relative to the total incremental displacement. If $\Delta \mathbf{a}^{(n)}$ is greater than $1 \%$ of the total incremental displacement, another iteration is performed. Only if both convergence requirements are satisfied can a solution be said to have converged for that load increment.

\section{Simulation Conditions}

3.1. FE Model. The commercial FEA software package, ABAQUS/Standard, has been used to develop the finite element model of flexible microrolling, as shown in Figure 2, and some basic geometric parameters of the model are given in Table 1.

The upper and lower rolls were treated as rigid bodies and meshed with 4-node 3D rigid quadrilateral elements (R3D4). The motion of nodes and elements that form the rigid rolls is governed by the rigid body reference nodes, which take the geometric centres of the upper and lower rolls, respectively. The shape of the rolls does not alter throughout the simulation process but can undergo rigid body motions pursuant to the boundary conditions applied at the reference nodes.

During the flexible microrolling process, the blank is pressed, leading to the thickness thinned and the longitudinal and latitudinal elongation. As the stress and strain distributions in the thickness direction have an impact on the springback after the whole forming process, solid elements must be deployed to directly indicate the initial thickness of the blank and also the variation of the blank thickness and the states of stress and strain along thickness direction after rolling. Thus, 8-node linear brick elements (C3D8R) were chosen to mesh the deformable blank. Reduced integration and enhanced hourglass control were employed for all the elements to decrease computation time and improve convergence [17].

The contact between the rolls and the blank was set as surface-to-surface contact, and two contact pairs were created, which are contact between the upper roll and the top
TABLE 1: Basic geometric parameters of the FE model.

\begin{tabular}{lc}
\hline Diameters of the rolls $(\mathrm{mm})$ & $D=25$ \\
Widths of the rolls $(\mathrm{mm})$ & $B=35$ \\
Length of the blank $(\mathrm{mm})$ & $L=40$ \\
Width of the blank $(\mathrm{mm})$ & $b=10$ \\
Thickness of the blank $(\mu \mathrm{m})$ & $t=500$ \\
\hline
\end{tabular}

TABLE 2: Loads and boundary conditions for phase I.

\begin{tabular}{cc}
\hline Position & Load and boundary condition \\
\hline \multirow{4}{*}{$A_{u}$} & $v_{x}=v_{y}=v_{z}=\omega_{x}=\omega_{y}=0$ \\
$\omega_{z}=-1.6 \mathrm{rad} / \mathrm{s}$ \\
$F_{y}=0$
\end{tabular}

TABLE 3: Loads and boundary conditions for phase II.

\begin{tabular}{cc}
\hline Position & Load and boundary condition \\
\hline$A_{u}$ & $v_{x}=v_{z}=\omega_{x}=\omega_{y}=0$ \\
$v_{y}= \pm \frac{\Delta h \omega_{z} D}{2 l}$ \\
$\omega_{z}=-1.6 \mathrm{rad} / \mathrm{s}$ \\
$F_{y}=-10 \mathrm{kN}$ \\
\hline & $v_{x}=v_{z}=\omega_{x}=\omega_{y}=0$ \\
$A_{l}$ & $v_{y}= \pm \frac{\Delta h \omega_{z} D}{2 l}$ \\
& $\omega_{z}=1.6 \mathrm{rad} / \mathrm{s}$ \\
& $F_{y}=10 \mathrm{kN}$ \\
\hline$\alpha$ & $v_{y}=0$
\end{tabular}

surface of the blank and contact between the lower roll and the bottom surface of the blank, respectively.

For the definition of the contact property, tangential behaviour was assigned adopting the penalty friction formulation. According to the engineering experience, the friction coefficient between rolls and blank is typically $0.1-0.2$, and the average friction coefficient 0.15 was selected in the cold rolling of stainless steel workpieces [13,18], so the friction coefficient 0.15 was selected in this study.

3.2. Loads and Boundary Conditions. The flexible microrolling process can be divided into two phases, that is, initial contact between the rolls and blank and flexible rolling. Different loads and boundary conditions were applied for different phases, as shown in Tables 2 and 3 , in which $A_{u}$ and $A_{l}$ are the geometric centres of the upper and lower rolls, separately, $\alpha$ is the middle surface of the blank to the thickness direction, $l$ is the length of the area of contact between the rolls and the blank, $\Delta h$ is the reduction amount, $v_{x}, v_{y}$, and $v_{z}$ are corresponding velocity components in directions $x, y$, and $z, \omega_{x}, \omega_{y}$, and $\omega_{z}$ are the angular velocity components of 


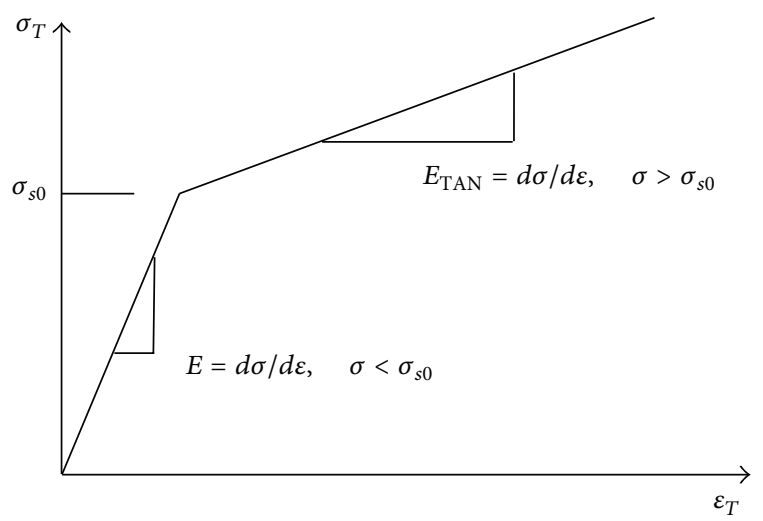

FIGURE 3: Bilinear isotropic hardening material model.

the rolls rotating about the axes $x, y$, and $z$, respectively, and $F_{y}$ is the concentrated force in direction $y$.

3.3. Yield Criterion. The von Mises yield criterion has been in use for the analysis of metal deformation in flexible microrolling process. It is expressed by

$$
f\left(\sigma_{i j}\right)-k_{0}=0
$$

where

$$
\begin{aligned}
f\left(\sigma_{i j}\right) & =\frac{1}{2} s_{i j} s_{i j}, \\
k_{0} & =\frac{1}{3} \sigma_{s 0}^{2}, \\
s_{i j} & =\sigma_{i j}-\sigma_{m} \delta_{i j}, \\
\sigma_{m} & =\frac{1}{3}\left(\sigma_{1}+\sigma_{2}+\sigma_{3}\right),
\end{aligned}
$$

where $\sigma_{s 0}$ is the initial yield stress of the material, $s_{i j}$ is the deviatoric stress tensor, $\sigma_{m}$ is the hydrostatic component of the stress, and $\delta_{i j}$ is the Kronecker delta. The relationship between $s_{i j}$ and effective stress, $\bar{\sigma}$, is given by

$$
\frac{1}{2} s_{i j} s_{i j}=\frac{\bar{\sigma}^{2}}{3}=J_{2} \text {, }
$$

where $J_{2}$ is the second stress invariant.

Equations (7)-(9) give

$$
\bar{\sigma}=\sigma_{s 0} .
$$

Therefore, the physical interpretation of von Mises criterion can be that plastic deformation begins when the effective stress is equal to the initial yield stress of the material.

3.4. Constitutive Relationship for Material. The material of the blank is stainless steel 304 and its mechanical properties are listed in Table 4, in which $\rho$ is the density, $E$ is Young's modulus, $v$ is Poisson's ratio, and $E_{\mathrm{TAN}}$ is the tangent modulus.

A bilinear isotropic hardening constitutive model depicted in Figure 3 is utilised for the blank as it is an approximation to the true stress-strain curve of the material and has been widely adopted in the nonlinear FEA [19-21].
TABLE 4: Mechanical properties of material.

\begin{tabular}{lccccc}
\hline Material & $\rho\left(\mathrm{kg} / \mathrm{m}^{3}\right)$ & $E(\mathrm{GPa})$ & $\nu$ & $\sigma_{s 0}(\mathrm{MPa})$ & $E_{\mathrm{TAN}}(\mathrm{MPa})$ \\
\hline Stainless steel & 8000 & 193 & 0.29 & 205 & 1630
\end{tabular}

TABLE 5: Number of meshes and computational time for six kinds of mesh sizes using hexahedron elements.

\begin{tabular}{lcccccc}
\hline Mesh size $(\mu \mathrm{m})$ & 500 & 250 & 150 & 100 & 50 & 25 \\
Number of meshes & 1,600 & 6,560 & 10,080 & 28,140 & 47,885 & 63,530 \\
Computation time $(\mathrm{h})$ & 2 & 9 & 14.5 & 41.5 & 72 & 96.5 \\
\hline
\end{tabular}

\section{Results and Discussion}

The simulation result with hexahedral mesh size $500 \mu \mathrm{m}$ is shown in Figure 4 . The springback in thickness direction, $\Delta t$, is calculated as $h+\Delta h-t$, where $h$ is the distance between the upper and lower surface nodes after deformation, $\Delta h$ is the designed reduction amount, and $t$ is the initial thickness of the blank.

The impact of mesh size on springback is discussed in Section 4.1 when the reduction is 20 and $50 \%$, respectively. As a comparison, tetrahedron elements (C3D10M) were employed for the blank and the results are analysed in Section 4.2. Moreover, the numerical integration of the finite element method is summarised in Section 4.3, which also gives an explanation for the difference of simulation results with usage of varying mesh types.

4.1. Effect of Mesh Size on Springback. The springback amount, $\Delta t$, is plotted versus mesh size in Figure 5 on the basis of the simulation outcomes. The preliminary mesh size is $500 \mu \mathrm{m}$, which means the blank consists of 1,600 hexahedron elements.

As can be seen in Figure 5, the springback dramatically decreases from the peak of about $9.0 \mu \mathrm{m}$ to the value of around $3.2 \mu \mathrm{m}$ and then slightly descends to approximately $2.0 \mu \mathrm{m}$ at the mesh size of 500,250 , and $25 \mu \mathrm{m}$, respectively, when the reduction is $20 \%$. For $50 \%$ reduction, the springback declines from the maximum of around $7.5 \mu \mathrm{m}$ to the minimum of about $1.2 \mu \mathrm{m}$ with the mesh size reducing from 500 to $25 \mu \mathrm{m}$, and a big drop of the springback from about 6.7 to $3.2 \mu \mathrm{m}$ shows up when the mesh size changes from 250 to $150 \mu \mathrm{m}$. For the whole range of the mesh size, the gap between the springback for $20 \%$ reduction and that for $50 \%$ reduction keeps within $3.5 \mu \mathrm{m}$, which is an accepted level in view of the material model in the simulation. As the springback variation is small after mesh size of $50 \mu \mathrm{m}$ for both reductions, $50 \mu \mathrm{m}$ can be regarded as the critical mesh size for the springback to slip into a stable stage in FEA simulation utilising hexahedron elements.

Table 5 exhibits the number of meshes and computational time for each mesh size. As can be observed in Table 5, the number of meshes and computational time both increase with the decrease of mesh size, which are 47,885 and $72 \mathrm{~h}$, respectively, at the critical mesh size of $50 \mu \mathrm{m}$.

4.2. Impact of Mesh Type on Springback. For the investigation of the impact of mesh type, tetrahedron elements were 


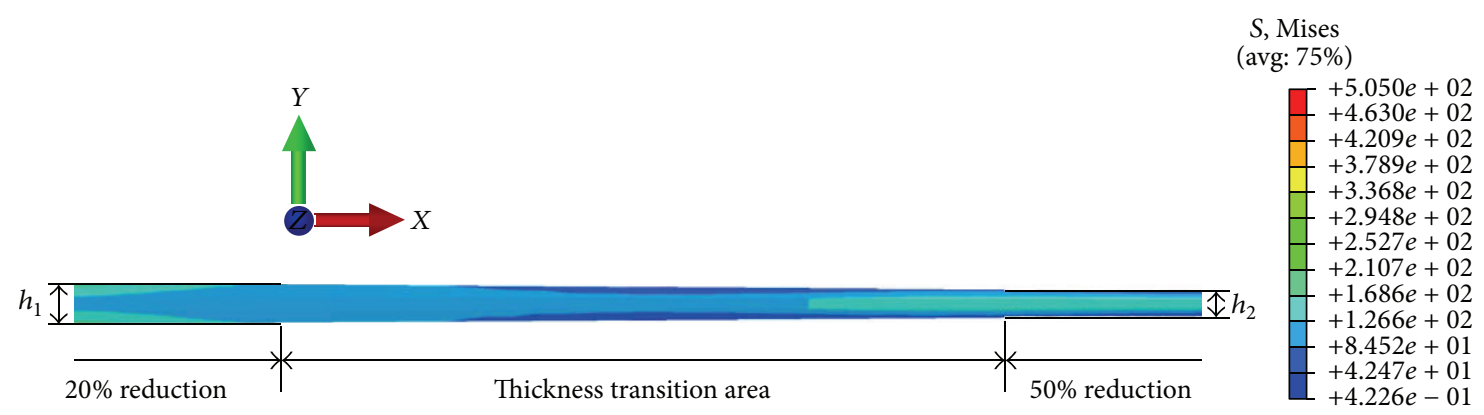

FIGURE 4: Simulation result with hexahedral mesh size $500 \mu \mathrm{m}$.

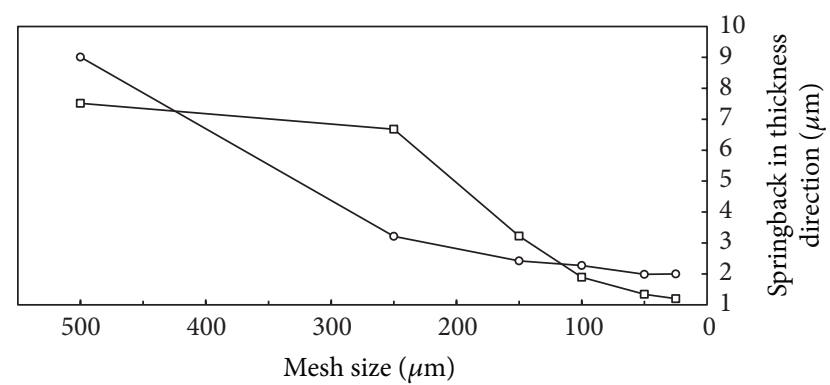

$\multimap-20 \%$ reduction

$\rightarrow-50 \%$ reduction

FIGURE 5: Springback in thickness direction versus mesh size using hexahedron elements.

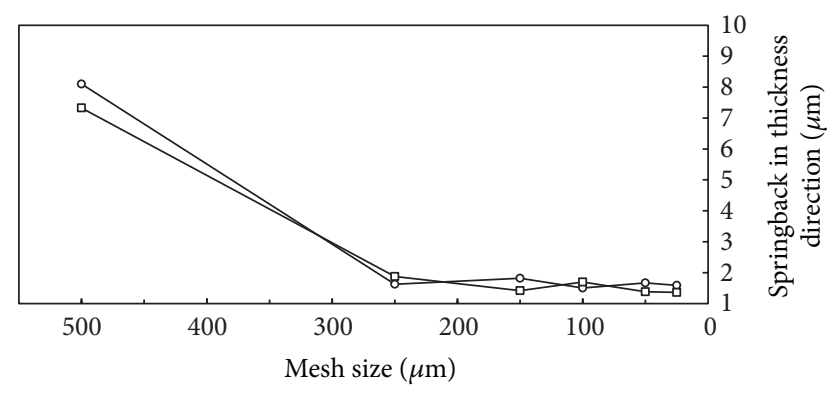

$\multimap 20 \%$ reduction

$\rightarrow-50 \%$ reduction

FIGURE 6: Springback in thickness direction versus mesh size using tetrahedron elements.

introduced for the blank and the size was chosen in line with that of hexahedron elements. Figure 6 shows the relationship between the springback and the mesh size in light of the simulation results.

It can be found out from Figure 6 that the trend and the amplitude of both curves are similar. The springback makes a sharp decrease from probably 8.1 to $1.6 \mu \mathrm{m}$ for $20 \%$ reduction and from roughly 7.4 to $1.9 \mu \mathrm{m}$ for $50 \%$ reduction when the mesh size is diminished to $250 \mu \mathrm{m}$. Although the springback amount fluctuates when the mesh size is less than $250 \mu \mathrm{m}$, the deviation is much smaller than that between mesh sizes of 500 and $250 \mu \mathrm{m}$ for both levels of reduction. Consequently, the mesh size of $250 \mu \mathrm{m}$ can be taken as the critical value for the
TABLE 6: Number of meshes and computational time for six sorts of mesh sizes using tetrahedron elements.

\begin{tabular}{lcccccc}
\hline Mesh size $(\mu \mathrm{m})$ & 500 & 250 & 150 & 100 & 50 & 25 \\
$\begin{array}{l}\text { Number of meshes } \\
\begin{array}{l}\text { Computation } \\
\text { time }(\mathrm{h})\end{array}\end{array}$ & 28 & 69.5 & 100.5 & 132.5 & 164 & 203.5 \\
\hline
\end{tabular}

springback amount to enter a steady phase in FEA simulation utilising tetrahedron elements.

After stabilisation, the springback remains at $1.5 \pm 0.5 \mu \mathrm{m}$ for the reduction of $20 \%$ and $50 \%$, which shows a good correlation with that based on hexahedron elements. The number of meshes and computational time utilising tetrahedral meshing are far more than those utilising hexahedral meshing for each mesh size, as displayed in Table 6. Nonetheless, the springback calculation achieved the same accuracy at the critical mesh size of $250 \mu \mathrm{m}$ with 4,495 fewer meshes and $2.5 \mathrm{~h}$ less computational time.

\subsection{Analysis of Numerical Integration in Flexible Microrolling} Simulation. Numerical integration requires, in general, that the integrand be evaluated at a finite number of points, called integration points, within the integration limits. Since the Gaussian quadrature is known to require the minimum number of integration points, the Gaussian quadrature formula is used to carry out the numerical integrations in the flexible microrolling simulation.

Consider the integrand $f(\xi, \eta, \zeta)$ defined by the natural coordinates $(-1 \leq \xi \leq 1,-1 \leq \eta \leq 1,-1 \leq \zeta \leq 1)$ in the three-dimensional space. In the Gaussian quadrature, the integration of $f(\xi, \eta, \zeta)$ can be evaluated by

$$
\begin{aligned}
& \int_{-1}^{1} \int_{-1}^{1} \int_{-1}^{1} f(\xi, \eta, \zeta) d_{\xi} d_{\eta} d_{\zeta} \\
& =\sum_{i=1}^{n} \sum_{j=1}^{m} \sum_{k=1}^{l} H_{i} H_{j} H_{k} f\left(\xi_{i}, \eta_{j}, \zeta_{k}\right),
\end{aligned}
$$

where $\xi_{i}, \eta_{j}$, and $\zeta_{k}$ are the coordinates of the integration points, $H_{i}, H_{j}$, and $H_{k}$ are the weight factors, and the summation is carried out over $n \times m \times l$ integration points. 
Usually, it is true that a higher-order integration is suggested in order to obtain accurate evaluations of integrands because some terms in the Gaussian quadrature are eliminated when fewer integration points are selected, which results in less accuracy [22]. For this reason, the difference resulting from different meshing technologies in flexible microrolling simulation fundamentally lies in the integration points that an element has. For reduced integration element C3D8R, there is only one integration point locating at the centre of the element. Though a small portion of the computer execution time is spent in performing this numerical integration, much error is introduced until 5 elements are adopted through the thickness. For full integration element $\mathrm{C} 3 \mathrm{D} 10 \mathrm{M}$, more than one integration point is distributed within the element, and hence accurate results can be achieved with merely two elements in the thickness direction.

\section{Conclusions}

This study shows the effects of mesh types on the accuracy of springback analysis in flexible microrolling process. Hexahedron and tetrahedron elements were involved in the finite element model in succession and six mesh sizes of $500,250,150,100,50$, and $25 \mu \mathrm{m}$ were separately selected for each mesh type. After stable convergence, the springback was found to keep at $1.5 \pm 0.5 \mu \mathrm{m}$ for both reductions 20 and $50 \%$ regardless of mesh type. Nevertheless, the critical mesh size of tetrahedron elements for a good convergence is $250 \mu \mathrm{m}, 5$ times coarser than that of hexahedron elements; namely, two divisions along the thickness direction are adequate to get satisfying results consuming less computing time. The optimum mesh type and mesh size for this study can be used as reference in creating other FE models for accurate and efficient numerical analyses.

Further work for this research can be (1) to evaluate the mesh effects on the accuracy of numerical simulation of flexible microrolling process through other respects, for instance, the surface asperity of the micro flexibly rolled blank, and (2) to develop mathematical models that reflect the relationship amongst mesh type, mesh size, and accuracy of results for acquiring accurate numerical results even when a coarser mesh with fewer integration points in it is used in presented FEA simulation.

\section{Conflict of Interests}

The authors declare that there is no conflict of interests regarding the publication of this paper.

\section{Acknowledgments}

The authors would like to thank the University of Wollongong and all the colleagues for their support to this study. The authors also would like to thank the Australian Research Council (ARC) support for the current study.

\section{References}

[1] Y. C. Shi, Z. X. Li, and H. Hao, "Mesh size effect in numerical simulation of blast wave propagation and interaction with structures," Transactions of Tianjin University, vol. 14, no. 6, pp. 396-402, 2008.

[2] C.-K. Choi and H.-G. Kwak, "The effect of finite element mesh size in nonlinear analysis of reinforced concrete structures," Computers \& Structures, vol. 36, no. 5, pp. 807-815, 1990.

[3] V. Cojocaru, Z. I. Korka, and C. O. Miclosina, "Influence of the mesh parameters on stresses and strains in FEM analysis of a gear housing," Anals of University "Eftimie Murgu" Resita, no. 2, pp. 47-52, 2013.

[4] S.-W. Kim, Y.-N. Kwon, and B.-S. Kang, "Influence of mesh density and element type on the accuracy of FE analysis of periodic cellular structures," Advanced Materials Research, vol. 445, pp. 583-588, 2012.

[5] I. M. Smith and R. Hobbs, "Finite element analysis of centrifuged and built-up slopes," Geotechnique, vol. 24, no. 4, pp. 531-559, 1974.

[6] M. Lai, S.-S. Feng, G.-Y. Huang, and T. Zhou, "Effects of mesh density and coupling control parameter in numerical simulation of fluid-structure interaction," in Proceedings of the International Conference on Computational and Information Sciences (ICCIS '11), pp. 1140-1143, IEEE, Chengdu, China, October 2011.

[7] M. F. S. Koslan, A. M. A. Zaidi, M. Z. Othman, S. Abdullah, and $S$. Thanakodi, "The effect of mesh sizing toward deformation result in computational dynamic simulation for blast loading application," Modern Applied Science, vol. 7, no. 7, pp. 23-28, 2013.

[8] M. V. Candal, R. A. Morales, and K. Gorrin, "Influence of mesh density on injection molding simulation results," Journal of Plastics Technology, vol. 3, pp. 1-10, 2007.

[9] C. H. Pham, S. Thuillier, and P.-Y. Manach, "2D springback and twisting of ultra-thin stainless steel U-shaped parts," Steel Research International, vol. 86, no. 8, pp. 861-868, 2015.

[10] W. L. Xu, C. H. Ma, C. H. Li, and W. J. Feng, "Sensitive factors in springback simulation for sheet metal forming," Journal of Materials Processing Technology, vol. 151, no. 1-3, pp. 217-222, 2004.

[11] P. A. Eggertsen, K. Mattiasson, and M. Larsson, "A comprehensive analysis of benchmark 4: pre-strain effect on springback of 2D draw bending," AIP Conference Proceedings, vol. 1383, no. 1, pp. 1064-1071, 2011.

[12] H. B. Xie, K. Manabe, T. Furushima, K. Tada, and Z. Y. Jiang, "An experimental and numerical investigation on micro rolling for ultra-thin strip," International Journal of Material Forming, 2015.

[13] H. B. Xie, K. Manabe, T. Furushima, K. Tada, and Z. Y. Jiang, "Deformation characterization of micro rolling for stainless steel foil," Procedia Engineering, vol. 81, pp. 179-184, 2014.

[14] Z. Y. Jiang and A. K. Tieu, "A 3-D finite element method analysis of cold rolling of thin strip with friction variation," Tribology International, vol. 37, no. 2, pp. 185-191, 2004.

[15] O. C. Zienkiewicz, The Finite Element Method in Engineering Science, McGraw-Hill Book Company, London, UK, 1971.

[16] Abaqus 6.11 Tutorials, Dassault Systèmes, Vélizy-Villacoublay, France, 2011. 
[17] F. F. Song, H. Yang, H. Li, M. Zhan, and G. J. Li, "Springback prediction of thick-walled high-strength titanium tube bending," Chinese Journal of Aeronautics, vol. 26, no. 5, pp. 1336-1345, 2013.

[18] H.-L. Yu, X.-H. Liu, H.-Y. Bi, and L.-Q. Chen, "Deformation behavior of inclusions in stainless steel strips during multi-pass cold rolling," Journal of Materials Processing Technology, vol. 209, no. 1, pp. 455-461, 2009.

[19] Z. Gao and Y. J. Bu, "Research on the wet shot blasting technology of strip steel surface topography and finite element simulation," in Proceedings of the International Conference on Materials, Environmental and Biological Engineering, pp. 595598, Guilin, China, March 2015.

[20] B. Gordan, A. Adnan, and M. A. K. Aida, "Soil saturated simulation in embankment during strong earthquake by effect of elasticity modulus," Modelling and Simulation in Engineering, vol. 2014, Article ID 191460, 7 pages, 2014.

[21] K. Tantikom, T. Aizawa, and T. Mukai, "Symmetric and asymmetric deformation transition in the regularly cell-structured materials. Part II: theoretical study," International Journal of Solids and Structures, vol. 42, no. 8, pp. 2211-2224, 2005.

[22] G. R. Liu and S. S. Quek, The Finite Element Method: A Practical Course, Butterworth-Heinemann, Oxford, UK, 2nd edition, 2013. 


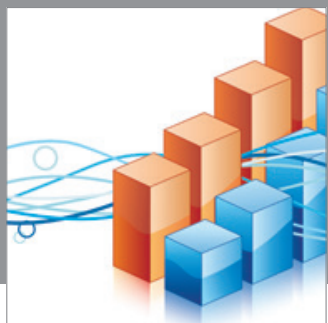

Advances in

Operations Research

mansans

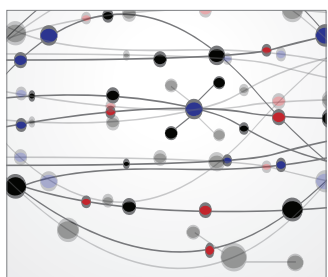

The Scientific World Journal
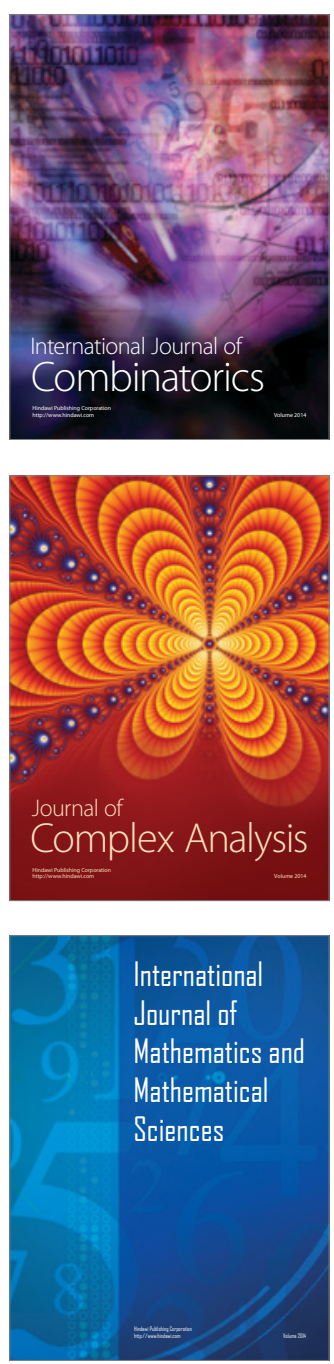
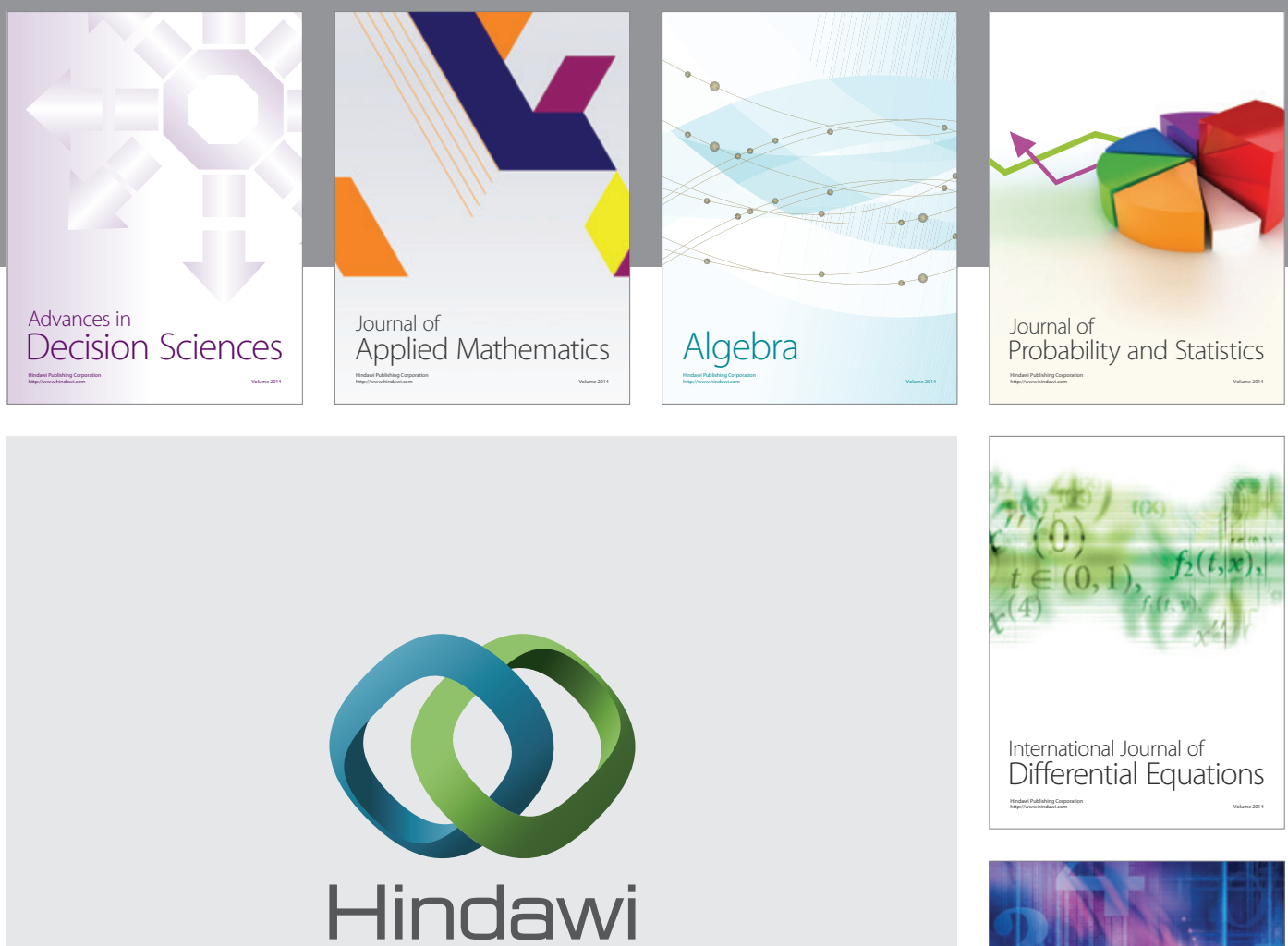

Submit your manuscripts at http://www.hindawi.com
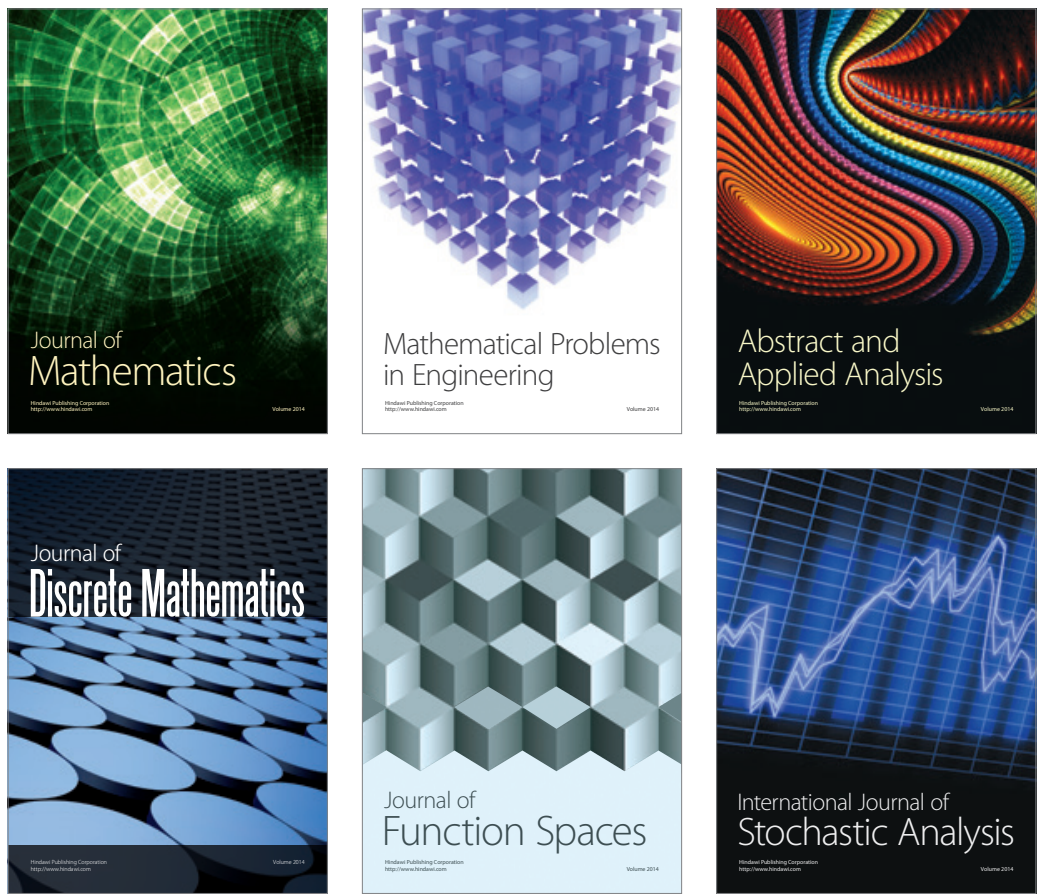

Journal of

Function Spaces

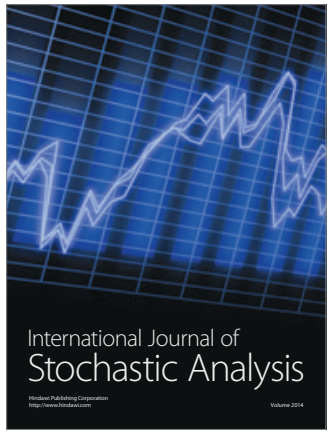

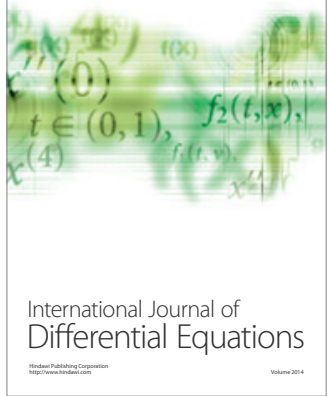
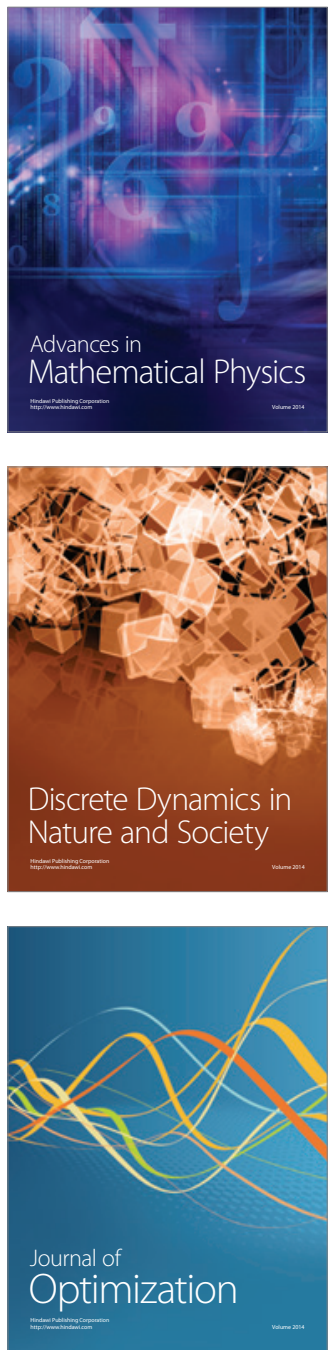